\title{
Erektile Dysfunktion als Warnsignal für drohende KHK ernst nehmen
}

— Vaskuläre Erkrankungen und Diabetes zeichnen für über 70\% der Fälle von erektiler Dysfunktion (ED) verantwortlich, wobei das Ausmaß mit dem Ausprägungsgrad der ED korreliert. Das verdeutlichte Prof. Dr. Graham Jackson vom London Bridge Hospital im Rahmen des 12. Kongresses der Europäischen Gesellschaft für Sexualmedizin (ESSM) in Lyon.

ED und koronare Herzkrankheit (KHK) haben die gleichen Risikofaktoren; gemeinsamer Nenner ist eine Endothel-Schädigung. Erektionsprobleme könnten somit als frühes Symptom einer generalisierten Gefäßschädigung betrachtet werden, so Jackson. In der COBRA-Studie hat sich ein starker zeitlicher Zusammenhang zwischen Erektionsproblemen und KHK gezeigt: Hier ging der Beginn der Erektionsstörung einer Angina pectoris in $93 \%$ der Fälle um im Mittel 24 Monate voraus.

Auch ohne kardiovaskuläre Erkrankung stellen Erektionsprobleme einen Prädiktor für nachfolgende kardiovaskuläre Ereignisse dar. In einer retrospektiven Studie lag das Risiko bei Männern mit ED doppelt so hoch wie in der Normalbevölkerung. Besonders bemerkenswert sei das siebenfach erhöhte Risiko bei Männern unter 40 Jahren, betonte Jackson. „Wir haben ein Zeitfenster von zwei bis fünf Jahren, um dieses zu reduzieren. Dazu ist Teamarbeit nötig zwischen dem Hausarzt, dem Diabetologen, dem Kardiologen und dem Urologen“, so Jackson. Gemäß den Princeton Consensus Leitlinien müssten Männer mit ED und fehlenden kardialen Symptomen so lange als vaskuläre Patienten gelten, bis das Gegenteil bewiesen sei, lautet sein Fazit.

Zur Behandlung der Erektionsprobleme stehen PDE-5-Hemmer wie Tadalafil (Cialis ${ }^{\circledR}$ ) zur Verfügung. Dieses Mittel, das sich auch bei Diabetikern und Patienten mit Herz- oder Gefäßerkrankungen als wirksam und gut verträglich erwiesen hat, ist seit September 2008 auch für die tägliche Dosierung mit $5 \mathrm{mg}$ zugelassen. Wie Prof. Dr. Hartmut Porst, künftiger Präsident der ESSM und niedergelassener Urologe aus Hamburg, berichtete, belegen mehrere Phase-III-Studien über einen Zeitraum von drei Monaten bis zu zwei Jahren, dass diese neue Option ebenso effektiv ist wie die bedarfsweise Anwendung von 20 mg. Dabei habe sich auch bei langfristiger Anwendung ein sehr günstiges Nebenwirkungsprofil ohne Anzeichen einer Tachyphylaxie gezeigt. 74,7 \% der Patienten und 73,3\% ihrer Partnerinnen waren mit der Behandlung zufrieden. Erhebungen hätten gezeigt, dass Frauen und Männer besonderen Wert auf spontanen Sex legen. „Diesen Patienten können wir mit der täglichen Gabe von Tadalafil nun helfen“, resümierte Porst. bm

Symposium „Sex, Heart, an Hormones Framing the Mosaic", im Rahmen des 12th Congress of the European Society for Sexual Medicine (ESSM), Lyon, 17. November 2009; Veranstalter: Lilly

\section{LHRH-Analoga bei Prostatakarzinom: Mehr Lebensqualität durch 6-Monats-Depot}

— Etwa die Hälfte der Prostatakarzinompatienten, die mit LHRH-Analoga (LHRHa) behandelt werden, wünschen sich eine Entlastung durch längere Injektionsintervalle. Dies ist das Ergebnis einer Umfrage, die zwischen August und Dezember 2008 in drei urologischen Netzwerken per Fragebogen durchgeführt wurde. Die andere Hälfte ist traditioneller und möchte weiterhin das bisherige Medikament oder die ständige Betreuung durch den Urologen. Ziel der Befragung war es, herauszufinden, wie betroffene Männer selbst zu ihrer Hormontherapie stehen. Von den 550 befragten Männern erhielten fast 90\% ein Drei-Monats-Depot. 19,7\% wurden seit einem Jahr behandelt, $37,8 \%$ seit zwei oder drei Jahren und $42,5 \%$ seit vier Jahren oder länger (darunter $4,1 \%$ seit mehr als 8 Jahren).

Die Auswertung ergab, dass fast die Hälfte der Teilnehmer ein 6-Monats-Depot bevorzugen würde, vor allem die Jüngeren unter ihnen (86\% der 40-49-Jährigen und 67\% der 50-59-Jährigen) und diejenigen, die bisher ein 3-Monats-Depot erhielten. Für das längere Depot sprach insbesondere, dass man mit zwei Spritzen pro Jahr seltener an die Krankheit erinnert würde und bei Reisen flexibler sei. Hauptargumente dagegen waren die Angst, seltener vom Arzt betreut zu werden, und der Wunsch, beim gewohnten Medikament zu bleiben. Je belastender die Patienten die regelmäßige Injektion empfanden, desto eher wünschten sie sich das 6-Monats-Depot.

Nach Dr. Frank Kahmann, Berlin, und Dr. Alwin Weber, Michelstadt, die die Untersuchung leiteten, sollten Patientenwünsche und -ängste bei der Therapieentscheidung berücksichtigt werden, denn die gemeinsam erarbeiteten und getroffenen Entscheidungen sind nicht nur besonders tragfähig, sondern erhöhen auch die Patientenbindung. Ein LHRHa-6-MonatsDepot ist ein zusätzliches Instrument für die individualisierte medikamentöse Tumortherapie, bei der der Urologe seinen Patienten kompetent begleiten sollte. Dem Wunsch vieler Patienten, beim gewohnten Medikament bleiben zu können, kommt entgegen, dass es jetzt ein neues, modernes und einfach anwendbares 6-MonatsDepot mit Leuprorelin in der Mikrokapselgalenik (Sixantone ${ }^{\circledR}$ ) gibt. $\mathrm{db}$

Nach Information von Takeda Pharma

\section{Männergesundheit im Blick}

_ Zusammen mit der Deutschen Gesellschaft für Andrologie (DGA) unterstützt Bayer Vital zum vierten Mal das Projekt eines jungen Forschers zur Männergesundheit mit einem Preis in Höhe von 10.000 Euro. Überreicht wurde der Preis am 14. November 2009 auf der DGA-Jahrestagung in Freiburg an Dr. Frank Tüttelmann, Münster. Er untersucht eine neue Hypothese zur Entstehung der Oligozoospermie. db

Nach Informationen von Bayer Vital 